\title{
Synthesizing Soot Particles in a Paraffin-Oil Flame
}

\author{
Masoud Darbandi' ${ }^{1,2}$, Majid Ghafourizadeh ${ }^{2}$, Gerry E. Schneider ${ }^{3}$ \\ ${ }^{1}$ Department of Aerospace Engineering, Sharif University of Technology \\ ${ }^{2}$ Institute for Nanoscience and Nano-technology, Sharif University of Technology, \\ Tehran, Iran \\ darbandi@sharif.edu; majid.ghafourizadeh@gmail.com \\ ${ }^{3}$ Department of Mechanical and Mechatronics Engineering, University of Waterloo \\ Waterloo, Ontario, Canada \\ gerry.schneider@uwaterloo.ca
}

\begin{abstract}
In this paper, we would like to know how to synthesize soot in a paraffin-oil flame. To do this, we use an in-house developed numerical code and simulate the soot formation in a turbulent paraffin-oil flame. However, it is necessary to evaluate first this code to ensure its reliable prediction of the soot formation and thermal behavior of the flame. So, we simulate a benchmark turbulent paraffin-oil flame and compare our obtained results against the available experimental data. The comparison insinuates the capability of current numerical code in correct predictions of both flame structure and pollution species. Then, a glass slide is installed inside the combustor. This disk-shaped glass slide is mounted at the centerline of the combustor and right at its axis. This combustor is then simulated and the aero-thermal and soot behaviors of paraffin-oil flame are investigated carefully. Our simulations show that the mounted glass slide can collect most of the soot generated in the flame. Our findings also indicate that the soot particles deposited in the back side of the glass slide have nearly the same micron sizes. This outcome is very attractive for the researchers and investigators, who seek approaches to synthesize the soot particles roughly with the same size.
\end{abstract}

Keywords: Soot particles, Soot synthesize, Paraffin-oil, Turbulent flame

\section{Nomenclature}

\begin{tabular}{|c|c|c|c|c|c|}
\hline$e$ & $=$ & effective magnitude & $v$ & $=$ & axial velocity component \\
\hline$f$ & $=$ & mixture fraction & $z$ & $=$ & axial component in cylindrical coordinates \\
\hline$f^{\prime \prime 2}$ & $=$ & mixture fraction variance & $R$ & $=$ & gas constant \\
\hline$h$ & $=$ & total enthalpy & $T$ & $=$ & temperature \\
\hline$m$ & $=$ & chemical species index counter & $\mathbf{V}$ & $=$ & velocity vector \\
\hline$m^{*}$ & $=$ & soot mass fraction & $W$ & $=$ & molecular weight \\
\hline$n$ & $=$ & total number of species & $Y$ & $=$ & mass fraction \\
\hline$n^{*}$ & $=$ & soot number density & $\varepsilon$ & $=$ & turbulence dissipation rate \\
\hline$p$ & $=$ & pressure & $k$ & $=$ & turbulence kinetic energy \\
\hline$r$ & $=$ & radial component in cylindrical coordinates & $\mu$ & $=$ & molecular viscosity coefficient \\
\hline$u$ & $=$ & radial velocity component & $\rho$ & $=$ & mixture density \\
\hline
\end{tabular}

\section{Introduction}

Many researches have been conducted in the past to synthesize carbon nano-particles in many different applications. One synthesizing approach has been to utilize the combustion for the generation of soot particles in different flames. So, we also adopt the same approach in this study to investigate synthesizing soot in a paraffin-oil flame.

To study the soot generation from flames, one robust tool has been the numerical analysis of such flames. It is because it can provide a deep understanding of all phenomena occurring during the processes. Indeed, the computational fluid dynamics CFD has been very effective and strong tool and it has been able to provide suitable numerical solutions for a wide range of problems. So, the numerical researchers have used CFD as their numerical tool to analyze different problems in their studies. We also adopt the numerical tool and simulate the soot formation in the paraffin-oil flame. 
In the current study, we numerically simulate a turbulent flame burning paraffin-oil. First, we need to know if our numerical tool is reliable for the prediction of thermal and soot behaviors of the flame. To do so, we simulate a benchmark turbulent kerosene flame and compare our obtained results with the experiment data available in literature. We continue our study further and simulate the test case considering a disc-type glass slide mounted inside the combustor on its axis. We observe the behavior of thermo/fluid flow and soot generated inside the combustors. Then, the observations are discussed suitably and the concluding remarks are highlighted and summarized at the end.

\section{The Governing Equations and the Computational Method}

Considering the different phenomena happening in the sooting turbulent reacting flow, the required governing equations include the equations of fluid flow, turbulence, combustion, energy transfer, and aerosol. They are given by [1-4]

$$
\begin{aligned}
& \nabla \cdot(\rho \mathbf{V})+\rho \frac{u}{r}=0 \\
& \nabla \cdot(\rho \mathbf{V} u)=-\frac{\partial p}{\partial r}+\nabla \cdot\left(\mu_{e} \nabla u\right)+\frac{\mu_{e}}{r} \frac{\partial u}{\partial r}-\mu_{e} \frac{u}{r^{2}} \\
& \nabla \cdot(\rho \mathbf{V} v)=-\frac{\partial p}{\partial z}+\nabla \cdot\left(\mu_{e} \nabla v\right)+\frac{\mu_{e}}{r} \frac{\partial v}{\partial r}-\rho \mathbf{g} \\
& \nabla \cdot(\rho \mathbf{V} k)=\nabla \cdot\left(\frac{\mu_{e}}{\sigma_{k}} \nabla k\right)+\frac{\mu_{e}}{\sigma_{k} r} \frac{\partial k}{\partial r}+G_{k}-\rho \varepsilon \\
& \nabla .(\rho \mathbf{V} \varepsilon)=\nabla \cdot\left(\frac{\mu_{e}}{\sigma_{\varepsilon}} \nabla \varepsilon\right)+\frac{\mu_{e}}{\sigma_{\varepsilon} r} \frac{\partial \varepsilon}{\partial r}+\frac{\varepsilon}{k}\left(c_{1} G_{k}-c_{2} \rho \varepsilon\right) \\
& \nabla \cdot(\rho \mathbf{V} f)=\nabla \cdot\left(\frac{\mu_{e}}{\sigma_{f}} \nabla f\right)+\frac{\mu_{e}}{\sigma_{f} r} \frac{\partial f}{\partial r} \\
& \nabla \cdot\left(\rho \mathbf{V} f^{\prime \prime 2}\right)=\nabla \cdot\left(\frac{\mu_{e}}{\sigma_{f}} \nabla f^{\prime \prime 2}\right)+\frac{\mu_{e}}{\sigma_{f} r} \frac{\partial f^{\prime \prime 2}}{\partial r}+c_{g} \mu_{e}(\nabla f)^{2}-\rho c_{\chi} \frac{\varepsilon}{k} f^{\prime \prime 2} \\
& \nabla \cdot(\rho \mathbf{V} h)=\nabla \cdot\left(\frac{\mu_{e}}{\sigma_{h}} \nabla h\right)+\frac{\mu_{e}}{\sigma_{h} r} \frac{\partial h}{\partial r}+q_{r a d} \\
& \nabla .\left(\rho \mathbf{V} m^{*}\right)=\nabla \cdot\left(\frac{\mu_{e}}{\sigma_{\text {soot }}} \nabla m^{*}\right)+\frac{\mu_{e}}{\sigma_{\text {soot }} r} \frac{\partial m^{*}}{\partial r}+C_{1} \rho^{2}\left(\frac{Y_{c_{2} H_{2}}}{W_{c_{2} H_{2}}}\right)^{2} \frac{Y_{c_{6} H_{5}}}{W_{c_{6} H_{5}}} \frac{W_{H_{2}}}{Y_{H_{2}}} e^{-4378 / T}+ \\
& C_{2} \rho^{2} \frac{Y_{C_{2} H_{2}}}{W_{c_{2} H_{2}}} \frac{Y_{c_{6} H_{6}}}{W_{c_{6} H_{6}}} \frac{Y_{c_{6} H_{5}}}{W_{c_{6} H_{5}}} \frac{W_{H_{2}}}{Y_{H_{2}}} e^{-6390 / T}+C_{3} \rho \frac{Y_{c_{2} H_{2}}}{W_{c_{2} H_{2}}} e^{-12100 / T}\left(\pi \rho N_{A} n^{*}\right)^{1 / 3}\left(\frac{6 \rho m^{*}}{\rho_{\text {soot }}}\right)^{2 / 3}- \\
& C_{4} \rho \frac{Y_{O H}}{W_{O H}} \sqrt{T}\left(\pi \rho N_{A} n^{*}\right)^{1 / 3}\left(\frac{6 \rho m^{*}}{\rho_{\text {soot }}}\right)^{2 / 3} \\
& \nabla .\left(\rho \mathbf{V} n^{*}\right)=\nabla \cdot\left(\frac{\mu_{e}}{\sigma_{n u c}} \nabla n^{*}\right)+\frac{\mu_{e}}{\sigma_{n u c} r} \frac{\partial n^{*}}{\partial r}+\frac{C_{1}}{M_{P}} \rho^{2}\left(\frac{Y_{c_{2} H_{2}}}{W_{c_{2} H_{2}}}\right)^{2} \frac{Y_{c_{6} H_{5}}}{W_{c_{6} H_{5}}} \frac{W_{H_{2}}}{Y_{H_{2}}} e^{-4378 / T}+ \\
& \frac{C_{2}}{M_{P}} \rho^{2} \frac{Y_{C_{2} H_{2}}}{W_{c_{2} H_{2}}} \frac{Y_{C_{6} H_{6}}}{W_{c_{6} H_{6}}} \frac{Y_{C_{6} H_{5}}}{W_{c_{6} H_{5}}} \frac{W_{H_{2}}}{Y_{H_{2}}} e^{-6390 / T}-\frac{1}{N_{A}} \sqrt{\frac{24 R T}{\rho_{\text {soot }} N_{A}}}\left(\frac{6 \rho m^{*}}{\pi \rho_{\text {soot }}}\right)^{1 / 6}\left(\rho N_{A} n^{*}\right)^{11 / 6}
\end{aligned}
$$


The mixture density is calculated via the equation of state $p=\rho R T \sum_{m=1}^{n} Y_{m} / W_{m}$. The current authors have developed an in-house computational fluid dynamics CFD code and employed it in numerical simulations of numerous combustion problems. The related literature shows that the numerical tool would provide reliable solutions in simulating of simulating of flow, turbulence, combustion, heat transfer, and soot aerosol. More details can be found in Refs. [3-8].

\section{The Test Case and Numerical Validation}

We chose the case studied by Young et al. [9] experimentally. The axisymmetric combustor is 0.6 and $0.0775 \mathrm{~m}$ in length and radius, respectively. The fuel nozzle has a diameter of $1.5 \mathrm{~mm}$. The gaseous pre-evaporated kerosene is injected via the fuel nozzle and the oxidizer, i.e. dry air, enters as a co-flow stream. The more details can be found in Ref. [9]. We use the CFD code to simulate the burner and then evaluate the obtained results comparing them with the experiment. Figure 1 shows that the results of current solution can suitably predict the flame structure considering the data of experiment.
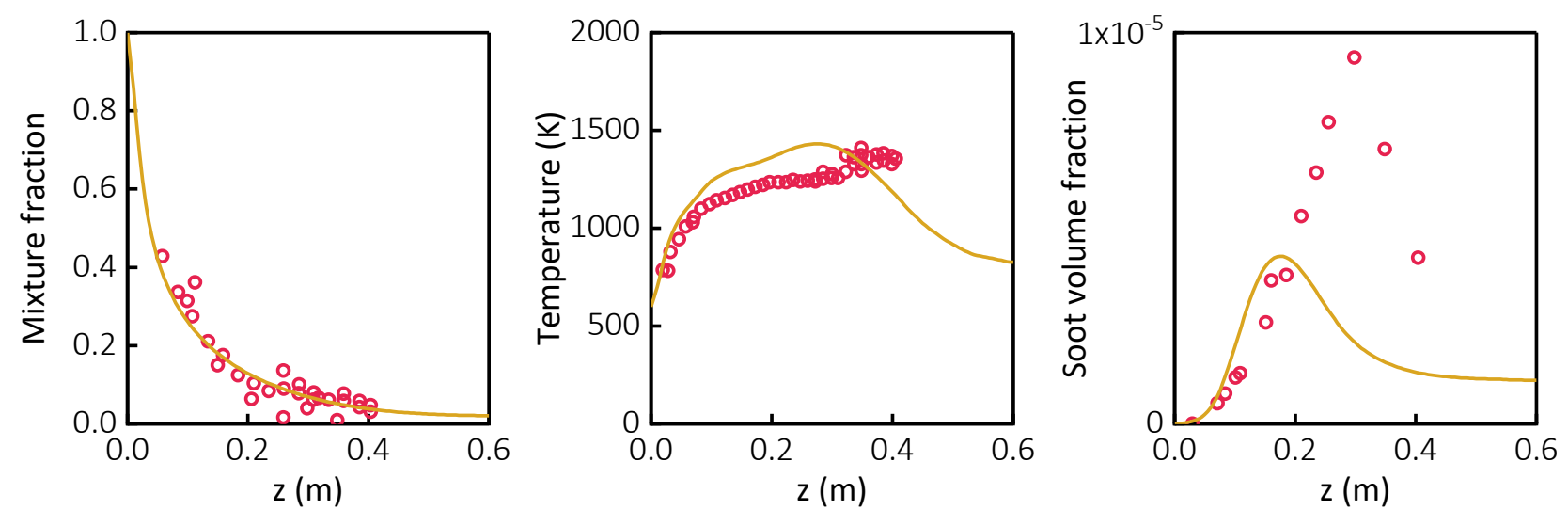

Fig. 1: The centerline distributions of quantities obtained in the current simulation and their comparisons against experiment.

\section{The Results and Discussion}

The next step, we install a glass slide inside the combustor. The glass slide has a disk shape. It is mounted at the center of combustor right at its axis. It is installed at a height of $0.2 \mathrm{~m}$ above the fuel nozzle. It has a radius of $0.07 \mathrm{~m}$ and a thickness of $1 \mathrm{~mm}$. Figure 2 shows the contours of stream function, temperature, soot volume fraction, and soot particles diameters inside the combustor.
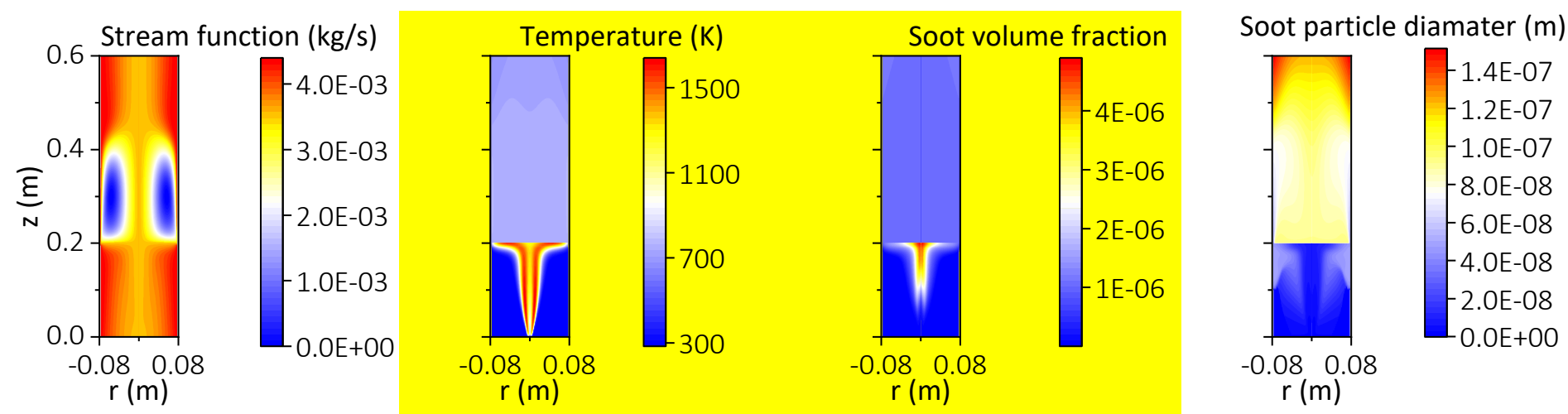

Fig. 2: The contours of stream function, temperature, soot volume fraction, and soot particles diameters inside the combustor. 
As seen in Fig. 2, the incoming flow arrives the glass slide, changes its direction towards the glass edge, where there is a gap between the glass slide and the combustor wall. The flow passes through the gap and reaches behind the back section of combustor. Passing through the gap, the flow velocity increases and the flow is injected into the space behind the glass slide. Such behavior causes the formation of a circulation zone behind the glass slide as observed in Fig. 2. The recirculation zone increases the mixing performance of gaseous mixture. It leads to a uniform-temperature gaseous mixture behind the glass slide. The well-mixed gaseous reacts together very well and form the combustion products. Apparently, the combustion by-products have time enough to reacts together effectively and to be burnt perfectly. It is anticipated that a homogeneous mixture would be achieved behind the glass slide; see the contour of soot volume fraction depicted in Fig. 2. Such behavior would results in soot particles with roughly the same size to be deposited on the back side of glass slide.

\section{Conclusion}

We simulated soot formation in a paraffin-oil flame. We adopted a numerical approach to perform the current study. First, the numerical tool was evaluated through simulating a benchmark test case with available experimental data. Our evaluation confirmed that the current tool would be capable of correct prediction of the proposed flame structure. We continued our activity and synthesize the soot particles in the flame. We installed a glass side inside the combustor over its axis to collect the soot generated from the flame. We simulated the new test case and observed the behavior of thermo-fluid flow behavior and the soot generated inside the combustor. Our observations indicated that the glass slide would collect most of the soot particles formed in the flame. Our findings also indicated that the soot would also deposit on the back side of the glass slide. They together showed that the glass slide could be used for synthesizing soot particles with nearly the same size in the flames. This can be considered as a remarkable outcome of the current study.

\section{References}

[1] J. H. Kent and D. Honnery, "Soot and Mixture Fraction in Turbulent Diffusion Flames," Combustion Science and Technology, vol. 54, no. 1-6, pp. 383-397, 1987.

[2] E. Ranzi, A. Frassoldati, A. Stagni, M. Pelucchi, A. Cuoci, and T. Faravelli, "Reduced Kinetic Schemes of Complex Reaction Systems: Fossil and Biomass-Derived Transportation Fuels," International Journal of Chemical Kinetics, vol. 46, no. 9, pp. 512-542, 2014.

[3] M. Darbandi, M. Ghafourizadeh, and M. Ashrafizaadeh, "Effect of Jet Impingement on Nano-Aerosol Soot Formation in a Paraffin-Oil Flame," in Mathematical and Computational Approaches in Advancing Modern Science and Engineering, J. Bélair, I. A. Frigaard, H. Kunze, R. Makarov, R. Melnik, and R. J. Spiteri, Eds. 1 ed. Switzerland: Springer International Publishing, 2016, pp. 89-99.

[4] M. Darbandi and M. Ghafourizadeh, "Numerical Study of Inlet Turbulators Effect on the Thermal Characteristics of a Jet Propulsion-Fueled Combustor and Its Hazardous Pollutants Emission," Journal of Heat Transfer, vol. 139, no. 6, pp. 1-12, 2017.

[5] M. Darbandi and M. Ghafourizadeh, "Extending a Hybrid Finite-Volume-Element Method to Solve Laminar Diffusive Flame," (in English), Numerical Heat Transfer, Part B: Fundamentals, vol. 66, no. 2, pp. 181-210, 2014.

[6] M. Darbandi and M. Ghafourizadeh, "Solving Turbulent Diffusion Flame in Cylindrical Frame Applying an Improved Advective Kinetics Scheme," (in English), Theoretical and Computational Fluid Dynamics vol. 29, no. 5-6, pp. 413431, 2015.

[7] M. Darbandi and M. Ghafourizadeh, "A New Bi-Implicit Finite Volume Element Method for Coupled Systems of Turbulent Flow and Aerosol-Combustion Dynamics," Journal of Coupled Systems and Multiscale Dynamics, vol. 4, no. 1, pp. 43-59, 2016.

[8] M. Darbandi and M. Ghafourizadeh, "Extending a Low-Order Upwind-Biased Scheme to Solve Turbulent Flames Using Detailed Chemistry Model," (in English), Numerical Heat Transfer, Part B: Fundamentals, vol. 73, no. 6, pp. 343-362, 2018. 
[9] K. J. Young, C. D. Stewart, and J. B. Moss, "Soot Formation in Turbulent Nonpremixed Kerosine-Air Flames Burning at Elevated Pressure: Experimental Measurement," Proceedings of the Combustion Institute, vol. 25, no. 1, pp. 609617, 1994. 